\title{
A Continuous State Feedback Controller Design for High-order Nonlinear Systems with Polynomial Growth Nonlinearities
}

\author{
Guo-Dong Zhao ${ }^{1} \quad$ Na Duan ${ }^{2}$ \\ ${ }^{1}$ School of Control Science and Engineering, Shandong University, Jinan 250061, China \\ ${ }^{2}$ School of Electrical Engineering and Automation, Jiangsu Normal University, Xuzhou 221116, China
}

\begin{abstract}
In this paper, we investigate the problem of global stabilization for a general class of high-order and non-smoothly stabilizable nonlinear systems with both lower-order and higher-order growth conditions. The designed continuous state feedback controller is recursively constructed to guarantee the global strong stabilization of the closed-loop system.
\end{abstract}

Keywords: Nonlinear systems, lower-order and high-order nonlinearities, state feedback, adding a power integrator, global strong stabilization.

\section{Introduction}

In this paper, we consider a class of high-order nonlinear systems

$$
\begin{aligned}
& \dot{x}_{i}(t)=x_{i+1}^{p_{i}}(t)+f_{i}(x(t), t), \quad i=1, \cdots, n-1 \\
& \dot{x}_{n}(t)=u^{p_{n}}(t)+f_{n}(x(t), t)
\end{aligned}
$$

where $x(t)=\left(x_{1}(t), \cdots, x_{n}(t)\right)^{\mathrm{T}} \in \mathbf{R}^{n}$ and $u(t) \in \mathbf{R}$ are the system state and control input, respectively. $p_{i} \in \mathbf{R}_{\text {odd }}^{\geqslant 1}=$ $\left\{\tilde{q} \epsilon_{+}: \tilde{q} \geqslant 1\right.$ is a ratio of odd integers $\}$ is said to be the high-order of system (1). When $p_{i} \geqslant 1$, system (1) is called high-order nonlinear system if at least one $p_{i}$ in system (1) satisfies $p_{i}>1$. The functions $f_{i}(x, t)(i=1, \cdots, n)$ : $\mathbf{R}^{n} \times \mathbf{R}_{+} \rightarrow \mathbf{R}$ are continuous in $(x, t)$ and $f_{i}(0, t)=0$.

In recent years, with the aid of the adding a power integrator scheme and backstepping method, the stabilization of the so-called high-order nonlinear system (1) has been an active area of research, and tremendous progress has been made toward the development of systematic design methodologies ${ }^{[1-3]}$. Following the state-feedback stabilization results ${ }^{[4,5]}$, for a family of systems (1) with uncontrollable/unobservable linearization, Yang et al. ${ }^{[6,7]}$ studied the robust output feedback stabilization with $f_{i}$ satisfying the higher-order growth condition and $p_{1}=\cdots=p_{n-1}=p$ being odd integers. Recently, by adopting a subtle homogeneous observer/controller construction and homogeneous domination design, the higher-order growth condition was replaced by higher-order homogeneous growth condition $^{[8-10]}$. For some special nonlinear systems with $p_{i}=1^{[11]}$, both linear and higher-order growth terms on $f_{i}$ were taken into account simultaneously.

It is well-known that the classical Lyapunov stability theory and the concepts, such as stability and asymptotic sta-

Manuscript received October 22, 2012; revised March 22, 2013

This work was supported by National Natural Science Foundation of China (Nos. 61273125 and 61104222), Specialized Research Fund for the Doctoral Program of Higher Education (No. 20103705110002), Program for the Scientific Research Innovation Team in Colleges and Universities of Shandong Province, Shandong Provincial Natural Science Foundation of China (No. ZR2012FM018), Natural Science Foundation of Jiangsu Province (No. BK2011205), and Natural Science Foundation of Jiangsu Normal University(No. 11XLR08). bility in the sense of Lyapunov, can only be applied to a nonlinear differential equation which has a unique solution. In [12], Kurzweil introduced the concepts related to the notion of global strong stability and Lyapunov stability theory in a continuous framework, without requiring uniqueness of the trajectories of a nonlinear differential equation. With the aid of Lyapunov stability theory for continuous system, Qian and Lin ${ }^{[13]}$ presented a new feedback design tool to solve global strong stabilization problem for system (1) with more general higher-order growth condition which is impossible to stabilize using any smooth feedback. Subsequently, they considered the stabilization problem for system (1) under the lower-order and linear growth condition, and only global strong stabilization via nonsmooth feedback was shown to be possible ${ }^{[14]}$. For more general nonlinear systems with both lower-order and higher-order growth conditions, Poiendo et al. ${ }^{[15-17]}$ further investigated the global strong stabilization. Naturally, one may ask the following important and interesting questions:

Whether the growth condition can be further relaxed? Under the weaker condition, can a continuous state feedback controller be designed?

In this paper, we will address the above problems and provide a satisfactory answer. For system (1), by relaxing the polynomial growth condition, combining the adding a power integrator scheme with backstepping method, skilfully constructing $\mathcal{C}^{1}$ Lyapunov functions, and employing a more flexible controller, we design a continuous state feedback controller to guarantee the global strong stabilization of the closed-loop system.

The paper is organized as follows. Section 2 provides the mathematical preliminaries. The design and analysis of the state feedback controller are given in Section 3, followed by a simulation example in Section 4 . Finally, Section 5 concludes this paper.

\section{Mathematical preliminaries}

The following notations will be used throughout the paper. $\mathbf{R}_{+}$denotes the set of all nonnegative real numbers, 
$\mathbf{R}^{n}$ denotes the real $n$-dimensional space, $\mathbf{R}_{\text {odd }}^{\geqslant 1}=\left\{\tilde{q} \in \mathbf{R}_{+}\right.$: $\tilde{q} \geqslant 1$ is a ratio of odd integers $\}$, and $\mathbf{R}_{\text {odd }}=\left\{\tilde{q} \in \mathbf{R}_{+}: \tilde{q}\right.$ is a ratio of odd integers $\}$. For a given vector or matrix $X$, $X^{\mathrm{T}}$ denotes its transpose, and $|X|$ is the Euclidean norm of vector $X . \mathcal{C}^{i}$ denotes the set of all functions with continuous $i$-th partial derivatives, $\mathcal{C}^{1,1}$ denotes the family of all functions which are $\mathcal{C}^{1}$ in the first argument for each fixed second argument, and $\mathcal{C}^{1}$ in the second argument for each fixed first argument. And $\mathcal{C}^{0}$ denotes the set of all continuous functions. To simplify the procedure, we sometimes denote $x(t)$ by $x$ for any variable $x(t)$.

Then, we give some definition and lemmas which will be frequently used in this paper for the design and analysis of controller.

Consider the following nonlinear systems

$$
\dot{x}=f(x, t), \quad x \in \mathbf{R}^{n}, \quad t \in \mathbf{R}
$$

with $f: \mathbf{R}^{n} \times \mathbf{R} \rightarrow \mathbf{R}^{n}$ being continuous in $(x, t)$ and $f(0, t)=0, \forall t \in \mathbf{R}$, And we do not need uniqueness of the trajectories of system (2) from any initial condition. Namely, system (2) may have infinite many solutions, which is why the notion of global strong stability is introduced in [12].

Definition $\mathbf{1}^{[12]}$. The trivial solution $x=0$ of system (2) is said to be globally strongly stable if there are two functions $B:(0,+\infty) \rightarrow(0,+\infty)$ and $T:(0,+\infty) \times(0,+\infty) \rightarrow$ $(0,+\infty)$ with $B$ being increasing and $\lim _{s \rightarrow 0} B(s)=0$, such that $\forall \alpha>0$ and $\forall \varepsilon>0$, for every solution $x(t)$ of system (2) defined on $\left[0, t_{1}\right), 0<t_{1} \leqslant+\infty$ with $|x(0)| \leqslant \alpha$, there is a solution $z(t)$ of system (2) defined on $[0,+\infty)$ satisfying: 1) $\left.z(t)=x(t), t \in\left[0, t_{1}\right), 2\right)|z(t)| \leqslant B(\alpha), \forall t \geqslant 0$, and 3$)$ $|z(t)| \leqslant \varepsilon, \forall t \geqslant T(\alpha, \varepsilon)$.

Lemma $\mathbf{1}^{[12]}$. We suppose there exist a $\mathcal{C}^{1,1}$ function $V(x, t): \mathbf{R}^{n} \times \mathbf{R} \rightarrow \mathbf{R}$, a $\mathcal{C}^{0}$ function $U_{1}: \mathbf{R}^{n} \rightarrow \mathbf{R}$, which is positive definite and proper, and $\mathcal{C}^{0}$ functions $U_{i}: \mathbf{R}^{n} \rightarrow \mathbf{R}$, $i=2,3$, which are positive definite, such that $U_{1}(x) \leqslant$ $V(x, t) \leqslant U_{2}(x), \frac{\partial V}{\partial t}+\frac{\partial V}{\partial x} f(x, t) \leqslant-U_{3}(x)$. Then, the trivial solution $x=0$ of system (2) is globally strongly stable.

Lemma $2^{[16]}$. Let $0 \leqslant \delta_{1} \leqslant \cdots \leqslant \delta_{n}$ be the real numbers and $c_{j}>0, j=1, \cdots, n$. Then, $\forall x \in \mathbf{R}, c_{1}|x|^{\delta_{1}}+c_{n}|x|^{\delta_{n}} \leqslant$ $\sum_{j=1}^{n} c_{j}|x|^{\delta_{j}} \leqslant\left(\sum_{j=1}^{n} c_{j}\right)\left(|x|^{\delta_{1}}+|x|^{\delta_{n}}\right)$.

Lemma $3^{[16]}$. For any $x, y \in \mathbf{R}$, if $p \geqslant 1$ is a constant, then $|x+y|^{p} \leqslant 2^{p-1}\left|x^{p}+y^{p}\right|$ and $(|x|+|y|)^{\frac{1}{p}} \leqslant|x|^{\frac{1}{p}}+|y|^{\frac{1}{p}} \leqslant$ $2^{\frac{p-1}{p}}(|x|+|y|)^{\frac{1}{p}}$. Moreover, if $p \in \mathbf{R}_{\text {odd }}^{\geqslant 1}$, then $|x-y|^{p} \leqslant$ $2^{p-1}\left|x^{p}-y^{p}\right|$ and $\left|x^{\frac{1}{p}}-y^{\frac{1}{p}}\right| \leqslant 2^{1-\frac{1}{p}}|x-y|^{\frac{1}{p}}$.

Lemma $4^{[16]}$. If $c$ and $d$ are positive constants, then for any positive number $\bar{\gamma},|x|^{c}|y|^{d} \leqslant \frac{c}{c+d} \bar{\gamma}|x|^{c+d}+$ $\frac{d}{c+d} \bar{\gamma}^{-\frac{c}{d}}|y|^{c+d}$.

Lemma 5 (Young's inequality). For any positive constant $\epsilon,|x||y| \leqslant \frac{\epsilon^{p}}{p}|x|^{p}+\frac{1}{q \epsilon^{q}}|y|^{q}$, where $p>1, q>1$, and $(p-1)(q-1)=1$.

\section{Controller design and analysis}

\subsection{Problem formulation}

The research purpose of this paper is to design a continuous state feedback controller for system (1) such that the closed-loop system is globally strongly stable. To achieve the control purpose, we need the following assumption.

Assumption 1. For $f_{i}(x, t)(i=1, \cdots, n)$ in system (1), there exist positive constants $c, r_{i}$ and $\tau_{i}$ such that

$$
\begin{aligned}
\left|f_{i}(x, t)\right| \leqslant & c\left(\left|x_{1}\right|^{\frac{1}{p_{1} \cdots p_{i-1}}}+\cdots+\left|x_{i-1}\right|^{\frac{1}{p_{i-1}}}+\left|x_{i}\right|+\right. \\
& \left.\left|x_{1}\right|^{\frac{r_{i}+\tau_{i}}{r_{1}}}+\cdots+\left|x_{i}\right|^{\frac{r_{i}+\tau_{i}}{r_{i}}}\right)
\end{aligned}
$$

where $r_{i}$ and $\tau_{i}$ are defined as

$$
\begin{aligned}
& r_{1}=1, \tau_{1} \geqslant 1, \quad r_{i+1} p_{i}=r_{i}+\tau_{i}, \\
& \tau_{k} \geqslant \max \left\{\left(\sum_{j=1}^{k-1} \frac{\tau_{j}}{r_{j}}\right) p_{1} \cdots p_{k-1} r_{k}, \tau_{k-1}\right\}, \\
& k=2, \cdots, n .
\end{aligned}
$$

Without loss of generality, we assume $\tau_{i}=\frac{n_{i}}{m_{i}}(i=$ $1, \cdots, n)$ with $n_{i}$ being an even integer and $m_{i}$ being an odd integer. Under this assumption, we know that $r_{1}, \cdots, r_{n+1} \in \mathbf{R}_{\text {odd }}$.

Remark 1. Let us discuss Assumption 1. Compared with [17], Assumption 1 is a more general and weaker condition. The assumption on $f_{i}$ in [17] is

$$
\begin{aligned}
\left|f_{i}(x, t)\right| \leqslant & c_{1}\left(\left|x_{1}\right|^{\frac{1}{p_{1} \cdots p_{i-1}}}+\cdots+\left|x_{i-1}\right|^{\frac{1}{p_{i-1}}}+\left|x_{i}\right|+\right. \\
& \left.\left|x_{1}\right|^{\frac{r_{i}+\tau}{r_{1}}}+\cdots+\left|x_{i}\right|^{\frac{r_{i}+\tau}{r_{i}}}\right), i=1, \cdots, n
\end{aligned}
$$

where $\tau$ is a positive constant. By using Lemma 2 and choosing appropriate positive constants $c$ and $\tau_{i}$, it can be obtained that

$$
\begin{gathered}
c_{1}\left(\left|x_{1}\right|^{\frac{1}{p_{1} \cdots p_{i-1}}}+\cdots+\left|x_{i-1}\right|^{\frac{1}{p_{i-1}}}+\left|x_{i}\right|+\right. \\
\left.\left|x_{1}\right|^{\frac{r_{i}+\tau}{r_{1}}}+\cdots+\left|x_{i}\right|^{\frac{r_{i}+\tau}{r_{i}}}\right) \leqslant \\
c\left(\left|x_{1}\right|^{\frac{1}{p_{1} \cdots p_{i-1}}}+\cdots+\left|x_{i-1}\right|^{\frac{1}{p_{i-1}}}+\left|x_{i}\right|+\right. \\
\left.\left|x_{1}\right|^{\frac{r_{i}+\tau_{i}}{r_{1}}}+\cdots+\left|x_{i}\right|^{\frac{r_{i}+\tau_{i}}{r_{i}}}\right) .
\end{gathered}
$$

Combining (6) with (5) leads to (3) in this paper. In other word, Assumption 1 includes the growth condition in [17].

On the other hand, when $\left\{\tau_{i}\right\}$ of Assumption 1 is any non-negative real number, we can still design the desired state feedback controller for system (1) without any modification, whose simple explanation is shown below. Since there exists $\bar{\tau}_{i}>0$ satisfying $\bar{\tau}_{i}=\frac{n_{i}}{m_{i}} \geqslant \tau_{i}$ with $n_{i}$ being an even integer and $m_{i}$ being an odd integer for $i=1, \cdots, n$, it is not difficult to deduce from Lemma 2 that

$$
\begin{aligned}
\left|f_{i}(x, t)\right| \leqslant & c_{2}\left(\left|x_{1}\right|^{\frac{1}{p_{1} \cdots p_{i-1}}}+\cdots+\left|x_{i-1}\right|^{\frac{1}{p_{i-1}}}+\left|x_{i}\right|+\right. \\
& \left.\left|x_{1}\right|^{\frac{r_{i}+\tau_{i}}{r_{1}}}+\cdots+\left|x_{i}\right|^{\frac{r_{i}+\tau_{i}}{r_{i}}}\right) \leqslant \\
& c_{3}\left(\left|x_{1}\right|^{\frac{1}{p_{1} \cdots p_{i-1}}}+\cdots+\left|x_{i-1}\right|^{\frac{1}{p_{i-1}}}+\left|x_{i}\right|+\right. \\
& \left.\left|x_{1}\right|^{\frac{r_{i}+\bar{\tau}_{i}}{r_{1}}}+\cdots+\left|x_{i}\right|^{\frac{r_{i}+\bar{\tau}_{i}}{r_{i}}}\right)
\end{aligned}
$$

where $c_{2}$ and $c_{3}$ are positive constants.

Thus, under the general condition on $f_{i}$, one can give the flexible controller design and analysis for system (1). 


\subsection{Design of state feedback controller}

Next, we construct a continuous state feedback controller for system (1) by combining the adding a power integrator with the backstepping technique.

Step 1. Let $\xi_{1}=x_{1}$ and $\mu=p_{1} \cdots p_{n} r_{n+1}$. Since the nonlinearities of system (1) consist of lower-order and higher-order parts, it is natural to construct a Lyapunov function with two parts: One is to deal with the lowerorder nonlinearities and the other is to deal with the higherorder nonlinearities. Now, construct the positive definite and proper Lyapunov function

$$
V_{1}=V_{L 1}+V_{H 1}=\frac{1}{2} \xi_{1}^{2}+\frac{r_{1}}{2 \mu-\tau_{1}} \xi_{1}^{\frac{2 \mu-\tau_{1}}{r_{1}}}
$$

whose time derivative along the trajectories of system (1) is

$$
\dot{V}_{1}=\left(\xi_{1}+\xi_{1}^{2 \mu-\tau_{1}-1}\right)\left(x_{2}^{p_{1}}+f_{1}(\cdot)\right) .
$$

By (3), Lemma 2 , and the definitions of $\mu, \tau_{1}$ and $r_{1}$ in (4), we have

$$
\begin{aligned}
& \left(\xi_{1}+\xi_{1}^{2 \mu-\tau_{1}-1}\right) f_{1}(\cdot) \leqslant \\
& \quad c\left(\left|\xi_{1}\right|+\left|\xi_{1}\right|^{2 \mu-\tau_{1}-1}\right) \times\left(\left|\xi_{1}\right|+\left|\xi_{1}\right|^{1+\tau_{1}}\right) \leqslant \\
& \quad a_{1,1}\left(\xi_{1}^{2}+\xi_{1}^{2 \mu}\right)
\end{aligned}
$$

where $a_{1,1}$ is a positive constant. Using (10), (9) turns into

$$
\dot{V}_{1} \leqslant\left(\xi_{1}+\xi_{1}^{2 \mu-\tau_{1}-1}\right) x_{2}^{p_{1}}+a_{1,1}\left(\xi_{1}^{2}+\xi_{1}^{2 \mu}\right) .
$$

Thus, it is not difficult to see that the first virtual control

$$
\begin{aligned}
& x_{2}^{*}=-\alpha_{1}\left(\xi_{1}+\xi_{1}^{\frac{r_{2} p_{1}}{r_{1}}}\right)^{\frac{1}{p_{1}}} \\
& \alpha_{1}=-\left(c_{1,1}+a_{1,1}\right)^{\frac{1}{p_{1}}}
\end{aligned}
$$

renders $\dot{V}_{1} \leqslant-c_{1,1}\left(\xi_{1}^{2}+\xi_{1}^{\frac{2 \mu}{r_{1}}}\right)+\left(\xi_{1}+\xi_{1}^{2 \mu-\tau_{1}-r_{1}}\right)\left(x_{2}^{p_{1}}-x_{2}^{* p_{1}}\right)$, where $c_{1,1}$ is a positive real number.

Step $\boldsymbol{i}(\boldsymbol{i}=\mathbf{2}, \cdots, \boldsymbol{n})$. At this step, we can obtain a property similar to Step 1, which is presented by the following lemma.

Lemma 6. Suppose at Step $i-1$, there are a $\mathcal{C}^{1}$ Lyapunov function $V_{i-1}: \mathbf{R}^{i-1} \rightarrow \mathbf{R}$, which is positive definite and proper, and a set of virtual controllers $x_{1}^{*}, x_{2}^{*}, \cdots, x_{i}^{*}$ defined by

$$
\begin{aligned}
x_{1}^{*}= & 0 \\
\xi_{1}= & x_{1}-x_{1}^{*} \\
x_{2}^{*}= & -\alpha_{1}\left(\xi_{1}+\xi_{1}^{\frac{r_{2} p_{1}}{r_{1}}}\right)^{\frac{1}{p_{1}}} \\
\xi_{2}= & x_{2}^{p_{1}}-x_{2}^{* p_{1}} \\
& \cdots \\
x_{i}^{*}= & -\alpha_{i-1}\left(\xi_{i-1}+\xi_{i-1}^{\frac{r_{i} p_{i-1}}{r_{i-1}}}\right)^{\frac{1}{p_{1} \cdots p_{i-1}}} \\
\xi_{i}= & x_{i}^{p_{1} \cdots p_{i-1}}-x_{i}^{* p_{1} \cdots p_{i-1}}
\end{aligned}
$$

with appropriate constants $\alpha_{j}>0(j=1, \cdots, i-1)$, such that

$$
\begin{aligned}
& \dot{V}_{i-1} \leqslant-\sum_{j=1}^{i-1} c_{i-1, j}\left(\xi_{j}^{2}+\xi_{j}^{\frac{2 \mu}{p_{1} \cdots p_{j-1} r_{j}}}\right)+ \\
& \quad\left(\xi_{i-1}^{2-\frac{1}{p_{1} \cdots p_{i-2}}}+\xi_{i-1}^{\frac{2 \mu-r_{i} p_{i-1}}{p_{1} \cdots p_{i-2} r_{i-1}}}\right) \times\left(x_{i}^{p_{i-1}}-x_{i}^{* p_{i-1}}\right)
\end{aligned}
$$

where $c_{i-1,1}, \cdots, c_{i-1, i-1}$ are positive constants. Then, there is a virtual control law $x_{i+1}^{*}=-\alpha_{i}\left(\xi_{i}+\xi_{i}^{\frac{r_{i+1} p_{i}}{r_{i}}}\right)^{\frac{1}{p_{1} \cdots p_{i}}}$, such that

$$
\begin{aligned}
\dot{V}_{i} \leqslant & -\sum_{j=1}^{i} c_{i, j}\left(\xi_{j}^{2}+\xi_{j}^{\frac{2 \mu}{p_{1} \cdots p_{j-1} r_{j}}}\right)+ \\
& \left(\xi_{i}^{2-\frac{1}{p_{1} \cdots p_{i-1}}}+\xi_{i}^{\frac{2 \mu-r_{i+1} p_{i}}{p_{1} \cdots p_{i-1} r_{i}}}\right) \times\left(x_{i+1}^{p_{i}}-x_{i+1}^{* p_{i}}\right)
\end{aligned}
$$

where the $i$-th Lyapunov function $V_{i}$ defined by

$$
\begin{aligned}
& V_{i}=V_{i-1}+V_{L i}+V_{H i} \\
& V_{L i}=\int_{x_{i}^{*}}^{x_{i}}\left(s^{p_{1} \cdots p_{i-1}}-x_{i}^{* p_{1} \cdots p_{i-1}}\right)^{2-\frac{1}{p_{1} \cdots p_{i-1}}} \mathrm{~d} s \\
& V_{H i}=\int_{x_{i}^{*}}^{x_{i}}\left(s^{p_{1} \cdots p_{i-1}}-x_{i}^{* p_{1} \cdots p_{i-1}}\right)^{\frac{2 \mu-r_{i+1} p_{i}}{p_{1} \cdots p_{i-1} r_{i}}} \mathrm{~d} s
\end{aligned}
$$

is $\mathcal{C}^{1}$, positive definite and proper, and $c_{i, 1}, \cdots, c_{i, i}$ are positive constants.

The proof of Lemma 6 can be seen in Appendix.

Hence, at Step $n$, choosing the state feedback controller of the form

$$
u^{p_{n}}=x_{n+1}^{* p_{n}}=-\alpha_{n}\left(\xi_{n}^{\frac{1}{p_{1} \cdots p_{n-1}}}+\xi_{n}^{\frac{r_{n+1} p_{n}}{p_{1} \cdots p_{n-1} r_{n}}}\right)
$$

yields

$$
\dot{V}_{n} \leqslant-\sum_{j=1}^{n} c_{n, j}\left(\xi_{j}^{2}+\xi_{j}^{\frac{2 \mu}{p_{1} \cdots p_{j-1} r_{j}}}\right)
$$

where $c_{n, 1}, \cdots, c_{n, n}$ are positive constants.

\subsection{Stability analysis}

We are now in the position to state the main theorem in this paper.

Theorem 1. If Assumption 1 holds for the high-order nonlinear system (1), under the state feedback controller (13) and (17), then the closed-loop systems (1), (13) and (17) are globally strongly stable.

Proof. Defining $U_{1}=\sum_{i=1}^{n} m_{i}\left(\left(x_{i}-x_{i}^{*}\right)^{2 p_{1} \cdots p_{i-1}}+\right.$ $\left.\left(x_{i}-x_{i}^{*}\right)^{\frac{2 \mu-r_{i+1} p_{i}}{r_{i}}+1}\right), U_{2}=\sum_{i=1}^{n} \bar{m}_{i}\left(\xi_{i}^{2}+\xi_{i}^{\frac{2 \mu-\tau_{i}}{p_{1} \cdots p_{i}-1 r_{i}}}\right)$, and $U_{3}=\sum_{j=1}^{i} c_{i, j}\left(\xi_{j}^{2}+\xi_{j}^{\frac{2 \mu}{p_{1} \cdots p_{j-1} r_{j}}}\right)$, together with Lemma 1, (18) and (24), imply that Theorem 1 holds.

Remark 2. We emphasize three points.

1) By introducing a more flexible definition of $\left\{\tau_{i}\right\}$ in Assumption 1 instead of $\tau$ in [17], we greatly enlarge the class of systems that we can deal with. Thus, the existing results in [17] can be viewed as a special case of this result. Furthermore, we can present a flexible controller design on 
state feedback for a more general class of systems compared with [17].

2) What should be stressed is that how to skillfully design the rigorous state feedback controller to dominate the nonlinearities and inequations is not a trivial work.

3) It is difficult to choose an appropriate Lyapunov function in the design and analysis of controller by combining the adding a power integrator scheme and backstepping approach. The high-order of system (1), and lower-order and higher-order nonlinearities in the system, and the repeated use of some inequalities will inevitably lead to the difficulty in choosing the Lyapunov function and controller.

\section{Simulation example}

Consider the nonlinear system

$$
\begin{aligned}
& \dot{x}_{1}=x_{2}+x_{1} \\
& \dot{x}_{2}=u^{3}+x_{1}+\left|x_{1}\right|^{\frac{1}{2}}\left|x_{2}\right|^{\frac{3}{2}} .
\end{aligned}
$$

One can choose $\tau_{1}=\frac{2}{3}, r_{2}=r_{1}+\tau_{1}=\frac{5}{3}, \tau_{2}=\frac{10}{3} \geqslant$ $\max \left\{\left(\frac{\tau_{1}}{r_{1}}\right) p_{1} r_{2}, \tau_{1}\right\}=\frac{16}{9}$, and $r_{3}=5$. Then, one gets $\left|f_{1}\right| \leqslant$ $\left|x_{1}\right|$ and $\left|f_{2}\right| \leqslant \frac{3}{2}\left(\left|x_{1}\right|+\left|x_{2}\right|^{3}\right)$. It is obvious that Assumption 1 holds.

Choose $\mu=p_{1} p_{2} r_{3}=5$. According to the design procedure in Section 3, one constructs the controller as

$$
\begin{aligned}
& \xi_{1}=x_{1} \\
& x_{2}^{*}=-3\left(\xi_{1}+\xi_{1}^{\frac{5}{3}}\right) \\
& \xi_{2}=x_{2}-x_{2}^{*} \\
& u=-92^{\frac{1}{3}}\left(\xi_{2}+\xi_{2}^{3}\right)^{\frac{1}{3}} .
\end{aligned}
$$

In the simulation, we choose the initial values $x_{1}(0)=1$ and $x_{2}(0)=-1$. Fig. 1 demonstrates the effectiveness of the controller.
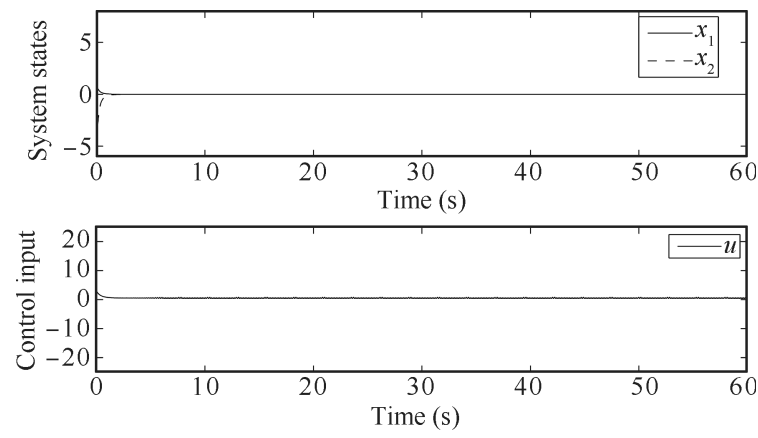

Fig. 1 The responses of the closed-loop systems (19) and (20)

\section{Conclusions}

In this paper, by combining the adding a power integrator method and backstepping approach, the continuous state feedback control problem is solved successfully for a class of high-order nonlinear systems under the polynomial growth condition.

Recently, for stochastic nonlinear systems and time-delay nonlinear systems, references [18-48] discussed the different control problems. However, all these references only considered the systems whose bounding condition is the allowed linear growth condition or higher-order growth condition. Therefore, an important problem is how to establish stochastic strong stability theory and give the design and analysis of the controller for stochastic nonlinear systems with both lower-order and higher-order nonlinearities.

\section{Appendix}

Proof of Lemma 6. By the definitions of $\mu, p_{i}, r_{i}$ in (4) and (16), it is easy to prove that $V_{L i}$ and $V_{H i}$ are $\mathcal{C}^{1}$. We can deduce from (13) and $\left|x^{\frac{1}{p}}-y^{\frac{1}{p}}\right| \leqslant 2^{1-\frac{1}{p}}|x-y|^{\frac{1}{p}}$ in Lemma 3 that

$$
\left|x_{i}-x_{i}^{*}\right| \leqslant 2^{1-\frac{1}{p_{1} \cdots p_{i-1}}}\left|\xi_{i}\right|^{\frac{1}{p_{1} \cdots p_{i-1}}} .
$$

Then, we divide the proof into two cases to prove that $V_{L i}$ and $V_{H i}$ are positive definite and proper.

Case 1. When $x_{i}^{*} \leqslant x_{i}$, using (16), (21), $p_{i} \in \mathbf{R}_{\text {odd }}^{\geqslant 1}$ and $|x-y|^{p} \leqslant 2^{p-1}\left|x^{p}-y^{p}\right|$ in Lemma 3 , we get

$$
\begin{aligned}
V_{L i}+ & V_{H i}=\int_{x_{i}^{*}}^{x_{i}}\left(s^{p_{1} \cdots p_{i-1}}-x_{i}^{* p_{1} \cdots p_{i-1}}\right)^{2-\frac{1}{p_{1} \cdots p_{i-1}}} \mathrm{~d} s+ \\
& \int_{x_{i}^{*}}^{x_{i}}\left(s^{p_{1} \cdots p_{i-1}}-x_{i}^{* p_{1} \cdots p_{i-1}}\right)^{\frac{2 \mu-r_{i+1} p_{i}}{p_{1} \cdots p_{i-1} r_{i}}} \mathrm{~d} s \geqslant \\
& 2^{1-p_{1} \cdots p_{i-1}}\left(\int_{x_{i}^{*}}^{x_{i}}\left(s-x_{i}^{*}\right)^{2 p_{1} \cdots p_{i-1}-1} \mathrm{~d} s+\right. \\
& \left.\int_{x_{i}^{*}}^{x_{i}}\left(s-x_{i}^{*}\right)^{\frac{2 \mu-r_{i+1} p_{i}}{r_{i}}} \mathrm{~d} s\right) \geqslant \\
& m_{i}\left(\left(x_{i}-x_{i}^{*}\right)^{2 p_{1} \cdots p_{i-1}}+\left(x_{i}-x_{i}^{*}\right)^{\frac{2 \mu-r_{i+1} p_{i}}{r_{i}}+1}\right)(22)
\end{aligned}
$$

where $m_{i}$ is a positive constant. By (16) and $\left|x^{\frac{1}{p}}-y^{\frac{1}{p}}\right| \leqslant$ $2^{1-\frac{1}{p}}|x-y|^{\frac{1}{p}}$ in Lemma 3, we have

$$
\begin{aligned}
V_{L i}+ & V_{H i} \leqslant\left(x_{i}^{p_{1} \cdots p_{i-1}}-x_{i}^{* p_{1} \cdots p_{i-1}}\right)^{2-\frac{1}{p_{1} \cdots p_{i-1}}} \times \\
& \left(x_{i}^{\frac{p_{1} \cdots p_{i-1}}{p_{1} \cdots p_{i-1}}}-x_{i}^{* \frac{p_{1} \cdots p_{i-1}}{p_{1} \cdots p_{i-1}}}\right)+ \\
& \left(x_{i}^{p_{1} \cdots p_{i-1}}-x_{i}^{* p_{1} \cdots p_{i-1}}\right)^{\frac{2 \mu-r_{i+1} p_{i}}{p_{1} \cdots p_{i-1} r_{i}}} \times \\
& \left(x_{i}^{\frac{p_{1} \cdots p_{i-1}}{p_{1} \cdots p_{i-1}}}-x_{i}^{* \frac{p_{1} \cdots p_{i-1}}{p_{1} \cdots p_{i-1}}}\right) \leqslant \\
& \bar{m}_{i}\left(\xi_{i}^{2}+\xi_{i}^{\frac{2 \mu-\tau_{i}}{p_{1} \cdots p_{i-1} r_{i}}}\right)
\end{aligned}
$$

where $\bar{m}_{i}$ is a positive constant.

Case 2. When $x_{i}^{*} \geqslant x_{i},(22)$ and (23) can be proved in a fashion similar to Case 1 .

From Cases 1 and 2, it follows that

$$
\begin{gathered}
\sum_{j=1}^{i} m_{j}\left(\left(x_{j}-x_{j}^{*}\right)^{2 p_{1} \cdots p_{j-1}}+\left(x_{j}-x_{j}^{*}\right)^{\frac{2 \mu-r_{j}+1 p_{j}}{r_{j}}+1}\right) \leqslant \\
V_{i} \leqslant \sum_{j=1}^{i} \bar{m}_{j}\left(\xi_{j}^{2}+\xi_{j}^{\frac{2 \mu-\tau_{j}}{p_{1} \cdots p_{j}-r_{j}}}\right) .
\end{gathered}
$$


(24) combined with (23) and the definitions of $\mu, r_{i}$ and $p_{i}$ in (4), implies that $V_{i}$ is positive definite and proper.

Now, we prove that (15) holds. By (1), (14) and (16), we have

$$
\begin{aligned}
\dot{V}_{i} & =\dot{V}_{i-1}+\sum_{j=1}^{i} \frac{\partial\left(V_{L i}+V_{H i}\right)}{\partial x_{j}}\left(x_{j+1}^{p_{j}}+f_{j}(\cdot)\right) \leqslant \\
& -\sum_{j=1}^{i-1} c_{i-1, j}\left(\xi_{j}^{2}+\xi_{j}^{\frac{2 \mu}{p_{1} \cdots p_{j-1} r_{j}}}\right)+\frac{\partial\left(V_{L i}+V_{H i}\right)}{\partial x_{i}} x_{i+1}^{p_{i}}+ \\
& \left(\xi_{i-1}^{2-\frac{1}{p_{1} \cdots p_{i-2}}}+\xi_{i-1}^{\frac{2 \mu-r_{i} p_{i-1}}{p_{1} \cdots p_{i-2} r_{i-1}}}\right)\left(x_{i}^{p_{i-1}}-x_{i}^{* p_{i-1}}\right)+ \\
& \frac{\partial\left(V_{L i}+V_{H i}\right)}{\partial x_{i}} f_{i}(\cdot)+\sum_{j=1}^{i-1} \frac{\partial\left(V_{L i}+V_{H i}\right)}{\partial x_{j}}\left(x_{j+1}^{p_{j}}+f_{j}(\cdot)\right) .
\end{aligned}
$$

Then, we concentrate on the last three terms on the righthand side of (25).

Before estimating the other terms, we present three useful inequations as follows. By (13), $|x+y|^{p} \leqslant 2^{p-1}\left|x^{p}+y^{p}\right|$ and $(|x|+|y|)^{\frac{1}{p}} \leqslant|x|^{\frac{1}{p}}+|y|^{\frac{1}{p}}$ in Lemma 3, we have

$$
\begin{aligned}
\left|x_{j}\right| \leqslant & \tilde{b}_{j, 1}\left(\left|\xi_{j}\right|^{\frac{1}{p_{1} \cdots p_{j-1}}}+\left|\xi_{j-1}\right|^{\frac{1}{p_{1} \cdots p_{j-1}}}+\right. \\
& \left.\left|\xi_{j-1}\right|^{\frac{r_{j}}{p_{1} \cdots p_{j-2} r_{j-1}}}\right) \\
\left|x_{1}\right| \leqslant & \left|\xi_{1}\right|
\end{aligned}
$$

where $\tilde{b}_{j, 1}$ is a positive constant, for $i \geqslant j \geqslant 2$. Using (3), (26), Lemma 2 and $|x+y|^{p} \leqslant 2^{p-1}\left|x^{p}+y^{p}\right|$ in Lemma 3, we obtain

$$
\left|f_{i}(\cdot)\right| \leqslant \hat{b}_{i} \sum_{j=1}^{i}\left(\left|\xi_{j}\right|^{\frac{1}{p_{1} \cdots p_{i-1}}}+\left|\xi_{j}\right|^{\frac{r_{i+1} p_{i}}{p_{1} \cdots p_{j}-r_{j}}}\right)
$$

where $\hat{b}_{i}$ is a positive constant. Putting (13) and $\left|x^{\frac{1}{p}}-y^{\frac{1}{p}}\right| \leqslant$ $2^{1-\frac{1}{p}}|x-y|^{\frac{1}{p}}$ in Lemma 3 together, we arrive at

$$
\left(x_{i}^{p_{i-1}}-x_{i}^{* p_{i-1}}\right) \leqslant 2^{1-\frac{1}{p_{1} \cdots p_{i-2}}}\left|\xi_{i}\right|^{\frac{1}{p_{1} \cdots p_{i-2}}} .
$$

Using (28), Lemmas 4 and $5\left(p=\frac{2 \mu}{2 \mu-r_{i} p_{i-1}}\right.$ and $q=$ $\frac{2 \mu}{r_{i} p_{i-1}}$ in Lemma 5), it is not difficult to verify that

$$
\begin{aligned}
& \left(\xi_{i-1}^{2-\frac{1}{p_{1} \cdots p_{i-2}}}+\xi_{i-1}^{\frac{2 \mu-r_{i} p_{i-1}}{p_{1} \cdots p_{i-2} r_{i-1}}}\right)\left(x_{i}^{p_{i-1}}-x_{i}^{* p_{i-1}}\right) \leqslant \\
& a_{i, 0}\left(\xi_{i-1}^{2}+\xi_{i-1}^{\frac{2 \mu}{p_{1} \cdots p_{i-2} r_{i-1}}}\right)+\bar{a}_{i, 0}\left(\xi_{i}^{2}+\xi_{i}^{\frac{2 \mu}{p_{1} \cdots p_{i-1} r_{i}}}\right)
\end{aligned}
$$

where $a_{i, 0}$ and $\bar{a}_{i, 0}$ are positive constants.

By (13), (16), (27), Lemmas 2, 4 and 5 with $p=$ $\frac{2 \mu}{2 \mu-r_{i+1} p_{i}}$ and $q=\frac{2 \mu}{r_{i+1} p_{i}}$, we get

$$
\begin{aligned}
& \frac{\partial\left(V_{H i}+V_{L i}\right)}{\partial x_{i}} f_{i}(\cdot)= \\
& \left(\xi_{i}^{2-\frac{1}{p_{1} \cdots p_{i-1}}}+\xi_{i}^{\frac{2 \mu-r_{i+1} p_{i}}{p_{1} \cdots p_{i-1} r_{i}}}\right) f_{i}(\cdot) \leqslant \\
& \hat{b}_{i}\left(\xi_{i}^{2-\frac{1}{p_{1} \cdots p_{i-1}}}+\xi_{i}^{\frac{2 \mu-r_{i+1} p_{i}}{p_{1} \cdots p_{i-1} r_{i}}}\right) \times \\
& \sum_{j=1}^{i}\left(\left|\xi_{j}\right|^{\frac{1}{p_{1} \cdots p_{i-1}}}+\left|\xi_{j}\right|^{\frac{r_{i+1} p_{i}}{p_{1} \cdots p_{j-1} r_{j}}}\right) \leqslant \\
& \sum_{j=1}^{i} a_{i, j}\left(\xi_{j}^{2}+\xi_{j}^{\frac{2 \mu}{p_{1} \cdots p_{j-1} r_{j}}}\right)
\end{aligned}
$$

where $a_{i, 1}, \cdots, a_{i, i}$ are positive constants.

At last, we concentrate on the term $\sum_{j=1}^{i-1} \frac{\partial\left(V_{L i}+V_{H i}\right)}{\partial x_{j}} \times$ $\left(x_{j+1}^{p_{j}}+f_{j}(\cdot)\right)$. For $j=1, \cdots, i-1$, by (13), (16) and the mean value theorem of integrals, we have

$$
\begin{aligned}
& \left(\frac{\partial V_{L i}}{\partial x_{j}}+\frac{\partial V_{H i}}{\partial x_{j}}\right)\left(x_{j+1}^{p_{j}}+f_{j}(\cdot)\right)= \\
& -\frac{\partial x_{i}^{* p_{1} \cdots p_{i-1}}}{\partial x_{j}}\left(\int_{x_{i}^{*}}^{x_{i}}\left(2-\frac{1}{p_{1} \cdots p_{i-1}}\right) \times\right. \\
& \left(s^{p_{1} \cdots p_{i-1}}-x_{i}^{* p_{1} \cdots p_{i-1}}\right)^{1-\frac{1}{p_{1} \cdots p_{i-1}}} \mathrm{~d} s+ \\
& \int_{x_{i}^{*}}^{x_{i}} \frac{2 \mu-r_{i+1} p_{i}}{p_{1} \cdots p_{i-1} r_{i}}\left(s^{p_{1} \cdots p_{i-1}}-\right. \\
& \left.\left.x_{i}^{* p_{1} \cdots p_{i-1}}\right)^{\frac{2 \mu-r_{i+1} p_{i}}{p_{1} \cdots p_{i-1} r_{i}}-1} \mathrm{~d} s\right)\left(x_{j+1}^{p_{j}}+f_{j}(\cdot)\right) \leqslant \\
& \tilde{b}_{j, 2}\left|\frac{\partial x_{i}^{* p_{1} \cdots p_{i-1}}}{\partial x_{j}}\right|\left|x_{j+1}^{p_{j}}+f_{j}(\cdot)\right| \times \\
& \left(\left|\xi_{i}\right|^{1-\frac{1}{p_{1} \cdots p_{i-1}}}+\left|\xi_{i}\right|^{\frac{2 \mu-r_{i+1} p_{i}}{p_{1} \cdots p_{i-1} r_{i}}-1}\right)\left|x_{i}-x_{i}^{*}\right|
\end{aligned}
$$

where $\tilde{b}_{j, 2}$ is a positive constant.

Next, we can prove that there exists a positive constant $\bar{l}_{i, j, 6}$ such that

$$
\begin{aligned}
& \left|\frac{\partial x_{i}^{* p_{1} \cdots p_{i-1}}}{\partial x_{j}}\right|\left|x_{j+1}^{p_{j}}+f_{j}(\cdot)\right| \leqslant \\
& \quad \bar{l}_{i, j, 6} \sum_{j=1}^{i}\left(\left|\xi_{j}\right|+\left|\xi_{j}\right|^{\frac{r_{i} p_{i-1} \cdots p_{1}+\tau_{i}}{p_{1} \cdots p_{j-1} r_{j}}}\right) .
\end{aligned}
$$

Indeed, by (4), (13), (26), (27), Lemmas 2-4 and 5 with 
$p=\frac{p_{1} \cdots p_{j-1} r_{j}+\tau_{j}}{p_{1} \cdots p_{j-1} r_{j}-r_{j}}$ and $q=\frac{p_{1} \cdots p_{j-1} r_{j}+\tau_{j}}{r_{j}+\tau_{j}}$, we get

$$
\begin{aligned}
& \left|\frac{\partial x_{i}^{* p_{1} \cdots p_{i-1}}}{\partial x_{j}}\right|\left|x_{j+1}^{p_{j}}+f_{j}(\cdot)\right| \leqslant \\
& l_{i, j, 1}\left(1+\left|\xi_{i-1}\right|^{\frac{\tau_{i-1}}{r_{i-1}}}\right) \cdots\left(1+\left|\xi_{j}\right|^{\frac{\tau_{j}}{r_{j}}}\right) \times \\
& \left|x_{j}\right|^{p_{1} \cdots p_{j-1}-1}\left(\left|x_{j+1}\right|^{p_{j}}+\left|f_{j}(\cdot)\right|\right) \leqslant \\
& l_{i, j, 2}\left(1+\left|\xi_{i-1}\right|^{\frac{\tau_{i-1}}{r_{i-1}}}\right) \cdots\left(1+\left|\xi_{j}\right|^{\frac{\tau_{j}}{r_{j}}}\right)\left(\left|\xi_{j}\right|+\left|\xi_{j-1}\right|+\right. \\
& \left.\left|\xi_{j-1}\right|^{\frac{r_{j} p_{j-1}}{r_{j-1}}}\right)^{1-\frac{1}{p_{1} \cdots p_{j-1}}}\left(\left|\xi_{j+1}\right|^{\frac{1}{p_{1} \cdots p_{j-1}}}+\left|\xi_{j}\right|^{\frac{1}{p_{1} \cdots p_{j-1}}}+\right. \\
& \left.\left|\xi_{j}\right|^{\frac{r_{j+1} p_{j}}{p_{1} \cdots p_{j-1} r_{j}}}+\sum_{k=1}^{j}\left(\left|\xi_{k}\right|^{\frac{1}{p_{1} \cdots p_{j-1}}}+\left|\xi_{k}\right|^{\frac{r_{j+1} p_{j}}{p_{1} \cdots p_{k-1} r_{k}}}\right)\right) \leqslant \\
& l_{i, j, 3}\left(1+\left|\xi_{i-1}\right|^{\frac{\tau_{i-1}}{r_{i-1}}}\right) \cdots\left(1+\left|\xi_{j}\right|^{\frac{\tau_{j}}{r_{j}}}\right) \times \\
& \left(\left|\xi_{j}\right|+\left|\xi_{j-1}\right|+\left|\xi_{j-1}\right|^{\frac{r_{j} p_{j-1}}{r_{j-1}}}\right)^{1-\frac{1}{p_{1} \cdots p_{j-1}}} \times \\
& \left(\left|\xi_{j+1}\right|^{\frac{1}{p_{1} \cdots p_{j-1}}}+\sum_{k=1}^{j}\left(\left|\xi_{k}\right|^{\frac{1}{p_{1} \cdots p_{j-1}}}+\left|\xi_{k}\right|^{\frac{r_{j+1} p_{j}}{p_{1} \cdots p_{k-1} r_{k}}}\right)\right) \leqslant \\
& l_{i, j, 4}\left(1+\left|\xi_{i-1}\right|^{\frac{\tau_{i-1}}{r_{i-1}}}\right) \cdots\left(1+\left|\xi_{j}\right|^{\frac{\tau_{j}}{r_{j}}}\right) \times \\
& \left(\left|\xi_{j+1}\right|+\sum_{k=1}^{j}\left(\left|\xi_{k}\right|+\left|\xi_{k}\right|^{\frac{p_{1} \cdots p_{j-1} r_{j}+\tau_{j}}{p_{1} \cdots p_{k-1} r_{k}}}\right)\right)= \\
& \underbrace{l_{i, j, 4}\left(1+\left|\xi_{i-1}\right|^{\frac{\tau_{i-1}}{r_{i-1}}}\right) \cdots\left(1+\left|\xi_{j}\right|^{\frac{\tau_{j}}{r_{j}}}\right)\left|\xi_{j+1}\right|}_{\Xi .1}+ \\
& l_{i, j, 4} \sum_{k=1}^{j}\left(\left|\xi_{k}\right|+\left|\xi_{k}\right|^{\frac{p_{1} \cdots p_{j-1} r_{j}+\tau_{j}}{p_{1} \cdots p_{k-1} r_{k}}}\right)\left(1+\left|\xi_{j}\right|^{\frac{\tau_{j}}{r_{j}}}\right) \times \\
& \left(1+\left|\xi_{j+1}\right|^{\frac{\tau_{j+1}}{r_{j+1}}}\right) \cdots\left(1+\left|\xi_{i-1}\right|^{\frac{\tau_{i-1}}{r_{i-1}}}\right)
\end{aligned}
$$

where $l_{i, j, 1}, l_{i, j, 2}, l_{i, j, 3}$ and $l_{i, j, 4}$ are positive constants. For $\Xi .1$ of (33), by (4), Lemmas 2 and 4, we obtain

$$
\begin{aligned}
& l_{i, j, 4}\left(1+\left|\xi_{i-1}\right|^{\frac{\tau_{i-1}}{r_{i-1}}}\right) \cdots\left(1+\left|\xi_{j}\right|^{\frac{\tau_{j}}{r_{j}}}\right)\left|\xi_{j+1}\right| \leqslant \\
& \bar{l}_{i, j, 1}\left(1+\sum_{k=j}^{i-1}\left|\xi_{k}\right|^{\sum_{m=j}^{i-1} \frac{\tau_{m}}{r_{m}}}\right)\left|\xi_{j+1}\right| \leqslant \\
& \bar{l}_{i, j, 2}\left(\left|\xi_{j+1}\right|+\left|\xi_{j+1}\right|^{1+\sum_{m=j}^{i-1} \frac{\tau_{m}}{r_{m}}}+\sum_{k=j}^{i-1}\left|\xi_{k}\right|^{1+\sum_{m=j}^{i-1} \frac{\tau_{m}}{r_{m}}}\right) \leqslant \\
& \bar{l}_{i, j, 3}\left(\sum_{j=1}^{i}\left(\left|\xi_{j}\right|+\left|\xi_{j}\right|^{\frac{r_{i} p_{i-1} \cdots p_{1}+\tau_{i}}{p_{1} \cdots p_{j-1} r_{j}}}\right)\right)
\end{aligned}
$$

where $\bar{l}_{i, j, 1}, \bar{l}_{i, j, 2}$ and $\bar{l}_{i, j, 3}$ are positive constants. To estimate the last term of (33), by using Lemmas 2 and 5 repeatedly, we observe from the definitions of $\mu, \tau_{i}$ in (4) that

$$
\begin{aligned}
& l_{i, j, 4} \sum_{k=1}^{j} \underbrace{\left(\left|\xi_{k}\right|+\left|\xi_{k}\right|^{\frac{p_{1} \cdots p_{j-1} r_{j}+\tau_{j}}{p_{1} \cdots p_{k-1} r_{k}}}\right)\left(1+\left|\xi_{j}\right|^{\frac{\tau_{j}}{r_{j}}}\right)}_{\Xi .2} \times \\
& \left(1+\left|\xi_{j+1}\right|^{\frac{\tau_{j+1}}{r_{j+1}}}\right) \cdots\left(1+\left|\xi_{i-1}\right|^{\frac{\tau_{i-1}}{r_{i-1}}}\right) \leqslant \\
& \bar{l}_{i, j, 4} \sum_{k=1}^{j}\left(\left|\xi_{k}\right|+\left|\xi_{k}\right|^{\frac{p_{1} \cdots p_{j} r_{j+1}+\tau_{j+1}}{p_{1} \cdots p_{k-1} r_{k}}}\right) \times \\
& \left(1+\left|\xi_{j+1}\right|^{\frac{p_{1} \cdots p_{j} \tau_{j+1}}{p_{1} \cdots p_{j} r_{j+1}}}\right) \cdots\left(1+\left|\xi_{i-1}\right|^{\frac{p_{1} \cdots p_{i-2} \tau_{i-1}}{p_{1} \cdots p_{i-2} r_{i-1}}}\right) \leqslant \\
& \bar{l}_{i, j, 5} \sum_{k=1}^{i-1}\left(\left|\xi_{k}\right|+\left|\xi_{k}\right|^{\frac{r_{i} p_{i-1} \cdots p_{1}+\tau_{i}}{p_{1} \cdots p_{k-1} r_{k}}}\right)
\end{aligned}
$$

where the first inequality is obtained by dealing with $\Xi .2\left(p=\frac{p_{1} \cdots p_{j} r_{j+1}+\tau_{j+1}}{p_{1} \cdots p_{j-1} \tau_{j}}\right.$ and $q=\frac{p_{1} \cdots p_{j} r_{j+1}+\tau_{j+1}}{p_{1} \cdots p_{j-1} r_{j}+\tau_{j+1}}$ in Lemma 5. After that, $\sum_{k=1}^{j}\left(\left|\xi_{k}\right|+\left|\xi_{k}\right|^{\frac{p_{1} \cdots p_{j} r_{j+1}+\tau_{j+1}}{p_{1} \cdots p_{k-1} r_{k}}}\right)(1+$ $\left.\left|\xi_{j+1}\right|^{\frac{p_{1} \cdots p_{j} \tau_{j+1}}{p_{1} \cdots p_{j} r_{j+1}}}\right) \quad\left(p=\frac{p_{1} \cdots p_{j+1} r_{j+2}+\tau_{j+2}}{p_{1} \cdots p_{j} \tau_{j+1}}\right.$ and $q=$ $\frac{p_{1} \cdots p_{j+1} r_{j+2}+\tau_{j+2}}{p_{1} \cdots p_{j} r_{j+1}+\tau_{j+2}}$ in Lemma 5$), \bar{l}_{i, j, 4}$ and $\bar{l}_{i, j, 5}$ are positive constants. Substituting (34) and (35) into (33) yields (32). Hence, by (21), (32), Lemmas 2, 4 and 5 with $p=\frac{2 \mu}{2 \mu-\tau_{i}-r_{i} p_{i-1} \cdots p_{1}}$ and $q=\frac{2 \mu}{p_{1} \cdots p_{i-1} r_{i}+\tau_{i}}$, there are positive constants $\bar{b}_{i, 1}, \cdots, \bar{b}_{i, i}, b_{i, 1}, \cdots, b_{i, i}$ and $\bar{l}_{i, j, 7}$ such that

$$
\begin{gathered}
\left(\frac{\partial V_{L i}}{\partial x_{j}}+\frac{\partial V_{H i}}{\partial x_{j}}\right)\left(x_{j+1}^{p_{j}}+f_{j}(\cdot)\right) \leqslant \\
\tilde{b}_{j, 2} \bar{l}_{i, j, 6}\left(\left|\xi_{i}\right|^{1-\frac{1}{p_{1} \cdots p_{i-1}}}+\left|\xi_{i}\right|^{\frac{2 \mu-r_{i+1} p_{i}}{p_{1} \cdots p_{i-1} r_{i}}-1}\right) \times \\
\left|x_{i}-x_{i}^{*}\right| \sum_{k=1}^{i}\left(\left|\xi_{k}\right|+\left|\xi_{k}\right|^{\frac{r_{i} p_{i-1} \cdots p_{1}+\tau_{i}}{p_{1} \cdots p_{k-1} r_{k}}}\right) \leqslant \\
\bar{l}_{i, j, 7}\left(\left|\xi_{i}\right|+\left|\xi_{i}\right|^{\frac{2 \mu-\tau_{i}-p_{1} \cdots p_{i-1} r_{i}}{p_{1} \cdots p_{i-1} r_{i}}}\right) \times \\
\sum_{k=1}^{i}\left(\left|\xi_{k}\right|+\left|\xi_{k}\right|^{\frac{r_{i} p_{i-1} \cdots p_{1}+\tau_{i}}{p_{1} \cdots p_{k-1} r_{k}}}\right) \leqslant \\
\sum_{j=1}^{i} \bar{b}_{i, j}\left(\xi_{j}^{2}+\xi_{j}^{\frac{2 \mu}{p_{1} \cdots p_{j-1} r_{j}}}\right) .
\end{gathered}
$$

Moreover,

$$
\begin{array}{r}
\sum_{j=1}^{i-1} \frac{\partial\left(V_{L i}+V_{H i}\right)}{\partial x_{j}}\left(x_{j+1}^{p_{j}}+f_{j}(\cdot)\right) \leqslant \\
\sum_{j=1}^{i} b_{i, j}\left(\xi_{j}^{2}+\xi_{j}^{\frac{2 \mu}{p_{1} \cdots p_{j-1} r_{j}}}\right) .
\end{array}
$$

With (25), (29), (30) and (36) in mind, it can be con- 
cluded that

$$
\begin{aligned}
\dot{V}_{i} \leqslant & -\sum_{j=1}^{i-1} c_{i-1, j}\left(\xi_{j}^{2}+\xi_{j}^{\frac{2 \mu}{p_{1} \cdots p_{j-1} r_{j}}}\right)+ \\
& a_{i, 0}\left(\xi_{i-1}^{2}+\xi_{i-1}^{\frac{2 \mu}{p_{1} \cdots p_{i-2} r_{i-1}}}\right)+\bar{a}_{i, 0}\left(\xi_{i}^{2}+\xi_{i}^{\frac{2 \mu}{p_{1} \cdots p_{i-1} r_{i}}}\right)+ \\
& \sum_{j=1}^{i} a_{i, j}\left(\xi_{j}^{2}+\xi_{j}^{\frac{2 \mu}{p_{1} \cdots p_{j}-1^{r_{j}}}}\right)+ \\
& \sum_{j=1}^{i} b_{i, j}\left(\xi_{j}^{2}+\xi_{j}^{\frac{2 \mu}{p_{1} \cdots p_{j-1} r_{j}}}\right)+ \\
& \left(\xi_{i}^{2-\frac{1}{p_{1} \cdots p_{i-1}}}+\xi_{i}^{\frac{2 \mu-r_{i+1} p_{i}}{p_{1} \cdots p_{i-1} r_{i}}}\right) x_{i+1}^{p_{i}}= \\
& -\sum_{j=1}^{i-1} c_{i, j}\left(\xi_{j}^{2}+\xi_{j}^{\frac{2 \mu}{p_{1} \cdots p_{j-1} r_{j}}}\right)+ \\
& \left(a_{i, i}+b_{i, i}+\bar{a}_{i, 0}\right)\left(\xi_{i}^{2}+\xi_{i}^{\overline{p_{1} \cdots p_{i-1} r_{i}}}\right)+ \\
& \left(\xi_{i}^{2-\frac{1}{p_{1} \cdots p_{i-1}}}+\xi_{i}^{\frac{2 \mu-r_{i+1} p_{i}}{p_{1} \cdots p_{i-1} r_{i}}}\right) x_{i+1}^{* p_{i}}+ \\
& \left(\xi_{i}^{2-\frac{1}{p_{1} \cdots p_{i-1}}}+\xi_{i}^{\frac{2 \mu-r_{i+1} p_{i}}{p_{1} \cdots p_{i-1} r_{i}}}\right)\left(x_{i+1}^{p_{i}}-x_{i+1}^{* p_{i}}\right) \\
& \\
&
\end{aligned}
$$

where $c_{i, j}=c_{i-1, j}-a_{i, j}-b_{i, j}, \quad j=1, \cdots, i-2, \quad c_{i, i-1}=$ $c_{i-1, i-1}-a_{i, 0}-a_{i, i-1}-b_{i, i-1}$. Finally, a virtual controller of the form $x_{i+1}^{* p_{1} \cdots p_{i}}=-\alpha_{i}^{p_{1} \cdots p_{i-1}}\left(\xi_{i}+\xi_{i}^{\frac{r_{i+1} p_{i}}{r_{i}}}\right), \quad \alpha_{i}=$ $\left(a_{i, i}+b_{i, i}+\bar{a}_{i, 0}+c_{i, i}\right)$, results in (15), which can be divided into the following two cases to prove.

1) When $\xi_{i}<0$, by $p_{i} \in \mathbf{R}_{\text {odd }}^{\geqslant 1},(|x|+|y|)^{\frac{1}{p}} \leqslant|x|^{\frac{1}{p}}+|y|^{\frac{1}{p}}$ in Lemma 3 and the definitions of $\mu$ and $r_{i}$, we have

$$
\begin{aligned}
& \left(\xi_{i}^{2-\frac{1}{p_{1} \cdots p_{i-1}}}+\xi_{i}^{\frac{2 \mu-r_{i+1} p_{i}}{p_{1} \cdots p_{i}-1 r_{i}}}\right) x_{i+1}^{* p_{i}}= \\
& -\left(\left(-\xi_{i}\right)^{2-\frac{1}{p_{1} \cdots p_{i-1}}}+\left(-\xi_{i}\right)^{\frac{2 \mu-r_{i+1} p_{i}}{p_{1} \cdots p_{i-1} r_{i}}}\right) \times \\
& \quad\left(a_{i, i}+b_{i, i}+\bar{a}_{i, 0}+c_{i, i}\right)\left(\left(-\xi_{i}\right)+\left(-\xi_{i}\right)^{\frac{r_{i+1} p_{i}}{r_{i}}}\right)^{\frac{1}{p_{1} \cdots p_{i-1}}} \leqslant \\
& -\left(a_{i, i}+b_{i, i}+\bar{a}_{i, 0}+c_{i, i}\right)\left(\xi_{i}^{2}+\xi_{i}^{\frac{2 \mu}{p_{1} \cdots p_{i-1} r_{i}}}\right) .
\end{aligned}
$$

2) When $\xi_{i} \geqslant 0$, similar to 1$)$, (38) also holds.

\section{References}

[1] W. Lin. http://nonlinear.cwru.edu/ linwei/, 2010.

[2] C. J. Qian. Global Synthesis of Nonlinear Systems with Uncontrollable Linearization, Ph. D. dissertation, Department of Electrical Engineering and Computer Science, Case Western Reserve University, USA, 2001.

[3] H. Lei. Universal Output Feedback Control of Nonlinear Systems, Ph. D. dissertation, Department of Electrical Engineering and Computer Science, Case Western Reserve University, USA, 2008.
[4] W. Lin, C. J. Qian. Adding one power integrator: A tool for global stabilization of high-order lower-triangular systems. Systems and Control Letters, vol. 39, no. 5, pp. 339$351,2000$.

[5] W. Lin, C. J. Qian. Adaptive regulation of high-order lowertriangular systems: An adding a power integrator technique. Systems and Control Letters, vol. 39, no. 5, pp. 353364, 2000.

[6] B. Yang, W. Lin. Robust output feedback stabilization of uncertain nonlinear systems with uncontrollable and unobservable linearization. IEEE Transactions on Automatic Control, vol. 50, no. 5, pp.619-630, 2005.

[7] N. Duan, F. N. Hu, X. Yu. An improved control algorithm for high-order nonlinear systems with unmodelled dynamics. International Journal of Automation and Computing, vol. 6, no. 3, pp. 234-239, 2009.

[8] J. Polendo, C. J. Qian. A generalized framework for global output feedback stabilization of genuinely nonlinear systems. In Proceedings of the 44th IEEE Conference on Decision and Control, and European Control Conference, IEEE, Seville, Spain, pp. 2646-2651, 2005.

[9] C. J. Qian. A homogeneous domination approach for global output feedback stabilization of a class of nonlinear systems. In Proceedings of the 2005 American Control Conference, IEEE, Portland, OR, USA, pp. 4708-4715, 2005.

[10] J. Polendo, C. J. Qian. A generalized homogeneous domination approach for global stabilization of inherently nonlinear systems via output feedback. International Journal of Robust and Nonlinear Control, vol. 17, no. 7, pp.605-629, 2007.

[11] V. Andrieu, L. Praly, A. Astolfi. Nonlinear output feedback design via domination and generalized weighted homogeneity. In Proceedings of the 45th IEEE Conference on Decision and Control, pp.6391-6396, 2006.

[12] J. Kurzweil. On the inversion of Lyapunov's second theorem on stability of motion. Czechoslovak Mathematical Journal, no. 81, pp. 217-259 and 455-473, 1956.

[13] C. J. Qian, W. Lin. A continuous feedback approach to global strong stabilization of nonlinear systems. IEEE Transactions on Automatic Control, vol. 46, no. 7, pp. 10611079, 2001.

[14] C. J. Qian, W. Lin. Recursive observer design, homogeneous approximation, and nonsmooth output feedback stabilization of nonlinear systems. IEEE Transactions on Automatic Control, vol. 51, no. 9, pp. 1457-1471, 2006.

[15] J. Polendo, C. J. Qian, H. Lei, W. Lin. A dual observer method for global stabilization of nonlinear systems with limited and uncertain information. In Proceedings of the American Control Conference, IEEE, New York, USA, pp. 2600-2605, 2007.

[16] H. Lei, W. Lin. Robust control of uncertain systems with polynomial nonlinearity by output feedback. International Journal of Robust and Nonlinear Control, vol.19, no. 6, pp. 692-723, 2009.

[17] H. Lei, W. Lin. Reduced-order observer, homogeneous domination and nonsmooth output feedback stabilization of nonlinear systems. In Proceedings of the 7th World Congress on Intelligent Control and Automation, IEEE, Chongqing, China, pp. 394-399, 2008

[18] Z. J. Wu, X. J. Xie, S. Y. Zhang. Stochastic adaptive backstepping controller design by introducing dynamic signal and changing supply function. International Journal of Control, vol. 79, no. 12, pp. 1635-1646, 2006.

[19] X. J. Xie, J. Tian. State-feedback stabilization for highorder stochastic nonlinear systems with stochastic inverse dynamics. International Journal of Robust and Nonlinear Control, vol. 17, no. 14, pp. 1343-1362, 2007. 
[20] J. Tian, X. J. Xie. Adaptive state-feedback stabilization for high-order stochastic non-linear systems with uncertain control coefficients. International Journal of Control, vol. 80, no. 9, pp. 1503-1516, 2007.

[21] Z. J. Wu, X. J. Xie, S. Y. Zhang. Adaptive backstepping controller design using stochastic small-gain theorem. $A u-$ tomatica, vol. 43, no. 4, pp. 608-620, 2007.

[22] J. Tian, X. J. Xie, C. H. Zhang. State feedback stabilization for high-order stochastic nonlinear systems with zero dynamics. Journal of Control Theory and Applications, vol. 6, no. 1, pp. 74-79, 2008.

[23] J. Tian, X. J. Xie. Adaptive state-feedback stabilization for more general high-order stochastic nonlinear systems. Acta Automatica Sinica, vol. 34, no. 9, pp. 1188-1192, 2008.

[24] W. Q. Li, X. J. Xie. Inverse optimal stabilization for stochastic nonlinear systems whose linearizations are not stabilizable. Automatica, vol. 45, no. 2, pp. 498-503, 2009.

[25] Z. J. Wu, X. J. Xie, P. Shi, Y. Q. Xia. Backstepping controller design for a class of stochastic nonlinear systems with Markovian switching. Automatica, vol.45, no.4, pp.9971004,2009

[26] X. J. Xie, J. Tian. Adaptive state-feedback stabilization of high-order stochastic systems with nonlinear parameterization. Automatica, vol. 45, no. 1, pp. 126-133, 2009.

[27] X. J. Xie, W. Q. Li. Output-feedback control of a class of high-order stochastic nonlinear systems. International Journal of Control, vol. 82, no. 9, pp. 1692-1705, 2009.

[28] X. Yu, N. Duan. Output feedback for stochastic nonlinear systems with unmeasurable inverse dynamics. International Journal of Automation and Computing, vol. 6, no. 4, pp. 391-394, 2009.

[29] L. Liu, X. J. Xie. Decentralized adaptive stabilization for interconnected systems with dynamic input-output and nonlinear interactions. Automatica, vol.46, no.6, pp.10601067, 2010.

[30] L. Liu, N. Duan, X. J. Xie. Output-feedback stabilization for stochastic high-order nonlinear systems with a ratio of odd integers power. Acta Automatica Sinica, vol. 36, no. 6, pp. 858-864, 2010.

[31] X. J. Xie, N. Duan. Output tracking of high-order stochastic nonlinear systems with application to benchmark mechanical system. IEEE Transactions on Automatic Control, vol. 55, no. 5, pp. 1197-1202, 2010.

[32] X. Yu, X. J. Xie, Y. Q. Wu. Further results on outputfeedback regulation of stochastic nonlinear systems with SiISS inverse dynamics. International Journal of Control, vol. 83, no. 10, pp. 2140-2152, 2010.

[33] X. Yu, X. J. Xie. Output feedback regulation of stochastic nonlinear systems with stochastic iISS inverse dynamics. IEEE Transactions on Automatic Control, vol. 55, no. 2, pp. 304-320, 2010.

[34] X. Yu, X. J. Xie, N. Duan. Small-gain control method for stochastic nonlinear systems with stochastic iISS inverse dynamics. Automatica, vol. 46, no. 11, pp. 1790-1798, 2010.

[35] X. J. Xie, N. Duan, X. Yu. State-feedback control of highorder stochastic nonlinear systems with SiISS inverse dynamics. IEEE Transactions on Automatic Control, vol. 56, no. 8, pp. 1921-1926, 2011.

[36] X. Yu, X. J. Xie, Y. Q. Wu. Decentralized adaptive outputfeedback control for stochastic interconnected systems with stochastic unmodeled dynamic interactions. International Journal of Adaptive Control and Signal Processing, vol. 25, no. 8, pp. 740-757, 2011.
[37] N. Duan, X. J. Xie. Further results on output-feedback stabilization for a class of stochastic nonlinear systems. IEEE Transactions on Automatic Control, vol. 56, no. 5, pp. 12081213, 2011.

[38] N. Duan, X. J. Xie, X. Yu. State feedback stabilization of stochastic nonlinear systems with SiISS inverse dynamics. International Journal of Robust and Nonlinear Control, vol. 21, no. 16, pp. 1903-1919, 2011.

[39] N. Duan, X. Yu, X. J. Xie. Output feedback control using small-gain conditions for stochastic nonlinear systems with SiISS inverse dynamics. International Journal of Control, vol. 84, no. 1, pp. 47-56, 2011.

[40] L. Liu, X. J. Xie. Output-feedback stabilization for stochastic high-order nonlinear systems with time-varying delay. Automatica, vol. 47, no. 12, pp. 2772-2779, 2011.

[41] Z. J. Wu, M. Y. Cui, X. J. Xie, P. Shi. Theory of stochastic dissipative systems. IEEE Transactions on Automatic Control, vol. 56, no. 7, pp. 1650-1655, 2011.

[42] W. Q. Li, X. J. Xie, S. Y. Zhang. Output-feedback stabilization of stochastic high-order nonlinear systems under weaker conditions. SIAM Journal on Control and Optimization, vol. 49, no. 3, pp. 1262-1282, 2011.

[43] L. Liu, X. J. Xie. State-feedback stabilization for stochastic high-order nonlinear systems with SISS inverse dynamics. Asian Journal of Control, vol. 14, no. 1, pp. 207-216, 2012.

[44] X. J. Xie, L. Liu. Further results on output feedback stabilization for stochastic high-order nonlinear systems with time-varying delay. Automatica, vol. 48, no. 10, pp. $2577-$ $2586,2012$.

[45] C. R. Zhao, X. J. Xie. Output feedback stabilization using small-gain method and reduced-order observer for stochastic nonlinear systems. IEEE Transactions on Automatic Control, vol. 58, no. 2, pp. 523-528, 2013.

[46] L. Liu, X. J. Xie. State feedback stabilization for stochastic feedforward nonlinear systems with time-varying delay. Automatica, vol. 49, no. 4, pp. 936-942, 2013.

[47] X. J. Xie, L. Liu. A homogeneous domination approach to state feedback of stochastic high-order nonlinear systems with time-varying delay. IEEE Transactions on Automatic Control, vol. 58, no. 2, pp. 494-499, 2013.

[48] Z. C. Zhang, Y. Q. Wu. Globally asymptotic stabilization for nonlinear time-delay systems with ISS inverse dynamics. International Journal of Automation and Computing, vol. 9, no. 6, pp. 634-640, 2012.

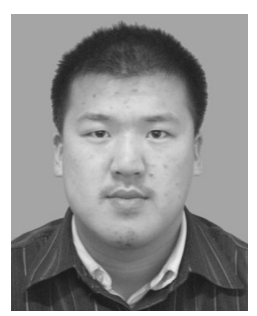

Guo-Dong Zhao received the M. Sc. degree from Qufu Normal University, China.

His research interests include nonlinear system controller design and stochastic nonlinear control.

E-mail: zgd_qufu@126.com (Corresponding author)

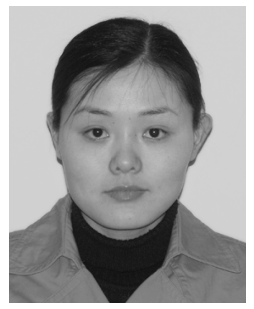

Na Duan received her Ph. D. degree from Institute of Automation, Qufu Normal University, China. Now she is an associate professor of Jiangsu Normal University, China.

Her research interests include high-order system controller design, nonlinear highorder system controller design, and stochastic nonlinear control.

E-mail: duanna08@163.com 